\title{
Isolation and Structure Determination of a Novel Complex of the Teicoplanin Family
}

\author{
Claudio Quarta, Angelo Borghi*, luigi Franco Zerilli, Maria Teresa De Pietro, \\ Pietro Ferrari, Aldo Trani and Gian Carlo lancini \\ Hoechst Marion Roussel, Lepetit Research Center, \\ Via R. Lepetit 34, 21040 Gerenzano, VA, Italy
}

(Received for publication December 4, 1995)

\begin{abstract}
A new teicoplanin-like antibiotic was discovered when using Actinoplanes teichomyceticus strain $3 / \mathrm{W}$, the fermentation medium containing Alburex, and the fermentation time 275 hours. The new product was separated from teicoplanin complex by polyamide resin chromatography and purified by Amberlite XAD-7 and affinity resin chromatographies. The structure was established on the basis of the physico-chemical characteristics of the complex and of its aglycone. The new structure is that of teicoplanin with a carbonyl group substituting for the $\mathrm{CHNH}_{2}$ group of amino acid 1 .

We hypothesize that the novel complex is a transformation product of teicoplanin due to a simple transamination reaction, as supported by its structure and by the concomitant decrease in teicoplanin concentration during its production.
\end{abstract}

During studies aimed at improving the production of teicoplanin by modification of the fermentation medium and of the culture conditions, the presence of a novel complex of antibiotics was noticed in the broth. The formation of this complex, named teico 2, showed a retention time higher than that of teicoplanin using reverse phase gradient HPLC analysis. Its presence depended both on the inclusion of Alburex $\mathrm{N}$ in the medium and on the increased time of fermentation. Conditions were found in which the novel complex was the major product, thus allowing its easy isolation with minimum teicoplanin content. The crude material was purified by affinity chromatography and further chromatographic procedures yielding a white amorphous powder, which was sufficiently pure for structural and biological activity studies.

We report here the fermentation conditions, the recovery procedure, and the results of the structure determination by NMR and MS spectroscopies on the intact molecules and on the aglycone obtained by acid hydrolysis. The teico 2 complex consisted of the analogues of teicoplanin's five main components in which the terminal $\mathrm{CH}-\mathrm{NH}_{2}$ is substituted by a $\mathrm{C}=\mathrm{O}$ group. The structure was confirmed by the synthesis of the teico 2 aglycone starting from teicoplanin aglycone.

\section{Experimental}

\section{Strain}

Actinoplanes teichomyceticus strain $3 / \mathrm{W}$, an improved teicoplanin producing strain, was used.

\section{Culture Media}

Agar medium SM: (g/liter) dextrose 10, Bacto-peptone 4, Bacto-yeast extract $4, \mathrm{MgSO}_{4} \cdot 7 \mathrm{H}_{2} \mathrm{O} 0.5, \mathrm{KH}_{2} \mathrm{PO}_{4} 2$, $\mathrm{K}_{2} \mathrm{HPO}_{4} 4$, Bacto-agar 20, deionized water up to.

Vegetative medium E/25: (g/liter) dextrose 25, meat extract 4 , yeast autolysate 1 , soybean meal 10 , peptone 4, $\mathrm{NaCl} 2.5, \mathrm{CaCO}_{3} 5$, deionized water up to.

Production medium T/2: (g/liter) dextrose 10, malt extract 20, yeast extract 4 , Alburex $\mathrm{N}$ (Roquette Frères) 10 , deionized water up to.

\section{Fermentation Conditions}

The strain was stored in lyophilized state. The slants were prepared from lyophilized vials on agar medium SM. The mycelium from a slant was homogenized in $10 \mathrm{ml}$ of saline and inoculated $(1 \% \mathrm{v} / \mathrm{v})$ in $500 \mathrm{-ml}$ Erlenmayer flasks each containing $100 \mathrm{ml}$ of medium $\mathrm{E} / 25$. The cultures were grown for 60 hours on a rotative shaker at $250 \mathrm{rpm}$ and at $28^{\circ} \mathrm{C}$, and used as inoculum $(0.14 \% \mathrm{v} / \mathrm{v})$ of a second pre-culture in a fermentor containing 8 liters of medium $\mathrm{E} / 25$. The culture was grown for 40 hours at $28^{\circ} \mathrm{C}$ with an air flow of 0.7 liter/ liter/minute and stirred at $600 \mathrm{rpm} .240 \mathrm{ml}$ of this culture was used as inoculum of 8 liters of medium $\mathrm{T} / 2$ and the fermentation was carried on at $28^{\circ} \mathrm{C}$, with an air flow of 0.7 liter $/$ liter $/$ minute and under a stirring of $600 \mathrm{rpm}$.

\section{Analytical HPLC}

Apparatus: two Waters $\bmod 510$ pumps, with the Automated Gradient Controller mod. 680; detector: Waters Lambda-Max, set at $254 \mathrm{~nm}$; column: Beckman Ultrasphere ODS, $4.6 \times 250 \mathrm{~mm}$; mobile phase: A: $0.2 \%$ $\mathrm{HCO}_{2} \mathrm{NH}_{4}-\mathrm{CH}_{3} \mathrm{CN} \quad(9: 1), \quad \mathrm{B}: \quad 0.2 \% \quad \mathrm{HCO}_{2} \mathrm{NH}_{4}-$ $\mathrm{CH}_{3} \mathrm{CN}$ (3:7); gradient: linear from $8 \% \mathrm{~B}$ to $40 \% \mathrm{~B}$ in 
40 minutes; flow rate: $1.5 \mathrm{ml} /$ minute.

\section{Column Chromatography}

Polyamide resin: the filtered broth was adsorbed on a polyamide resin (volume ratio $8: 1$ ), which was washed with $0.2 \mathrm{M}$ sodium phosphate $\mathrm{pH} 7.7$ and then with $0.2 \mathrm{M}$ sodium phosphate brought to $\mathrm{pH} 4.9$ with $\mathrm{NaOH}$. Elution of teicoplanin was performed with a $80 / 20$ mixture of $0.2 \mathrm{M}$ sodium phosphate $\mathrm{pH} 4.9$ /acetone. The elution of teico 2 was obtained with a $1 / 1$ mixture of $0.2 \mathrm{M}$ sodium phosphate $\mathrm{pH} 4.9 /$ acetone.

Amberlite XAD-7: the acetone in the eluate containing teico 2 was removed under vacuum and the aqueous solution was adsorbed on an Amberlite XAD-7 resin (volume ratio 15:1). The resin was washed with water and eluted with a $1 / 1$ mixture of acetone/water. Precipitation was obtained by adjusting the $\mathrm{pH}$ to 7.3 and adding 10 volumes of acetone.

Affinity resin: the crude material was dissolved in water and passed through an affinity column of Sepharoseacyl-D-alanyl-D-alanine resin $(50 \mathrm{ml} / \mathrm{g})^{11}$. The column was washed with 2 volumes of a $0.2 \% \mathrm{HCO}_{2} \mathrm{NH}_{4}$ solution and eluted with an aqueous solution of $\mathrm{NH}_{4} \mathrm{OH}$ $(1 \%, \mathrm{w} / \mathrm{v})$. The fractions $(10 \mathrm{ml}$ each) were pooled according to their composition.

Ultrafiltration: the pool containing teico 2 was neutralized with $\mathrm{HCO}_{2} \mathrm{H}$ and concentrated in a $62 \mathrm{~mm}$ Amicon Stirred Cell, supporting a Diaflo ultrafiltration membrane with a nominal molecular limit of $1000 \mathrm{Da}$. The retentate was then lyophilized.

\section{Fast Atom Bombardment Mass Spectrometry}

FAB-MS was obtained with a Kratos MS-50 instrument in positive ion mode using $\alpha$-thioglycerol containing $0.6 \% \mathrm{w} / \mathrm{v}$ of acetic acid as matrix. For FAB $\mathrm{Xe}$ gas was used, the gun was operated at $6 \mathrm{kV}$ potential. Calibration was obtained using a mixture of CsI and $\mathrm{NaI}^{2}$.

\section{Proton NMR Spectroscopy}

Proton NMR spectra were recorded in DMSO- $d_{6}$ solution at $22^{\circ} \mathrm{C}$ on a Bruker $\mathrm{AM}-500$ or a Bruker AM-250 spectrometer, both equipped with an Aspect 3000 computer. The phase-sensitive double quantum filter ${ }^{1} \mathrm{H}_{-}{ }^{1} \mathrm{H}-\mathrm{COSY}$ spectra were run using time-proportional-phase-increment in $\mathrm{fl}^{3)}$.

\section{UV Spectroscopy}

The UV spectra were recorded with a Perkin-Elmer spectrophotometer mod. 320 in methanol/water $(1 / 24$ $\mathrm{v} / \mathrm{v}$ ) solutions at various $\mathrm{pH}$ values.

\section{Synthesis of Teico 2 Aglycone}

Teicoplanin aglycone $\left(3 \mathrm{~g}\right.$, containing $\left.10 \% \mathrm{H}_{2} \mathrm{O}\right)$ was dissolved in $n$-butanol and the solvent evaporated to dryness under vacuum; this operation was repeated twice to remove the crystallization water. The aglycone was suspended under stirring in DMF $(40 \mathrm{ml})$ and a solution of mesityl-glyoxal ${ }^{4)}(480 \mathrm{mg})$ in DMF $(10 \mathrm{ml})$ was slowly added, keeping the temperature at $5^{\circ} \mathrm{C}$. After 10 minutes, the reaction was allowed to reach room temperature and the aglycone went in solution ${ }^{5)}$. The mixture was stirred overnight and the reddish solution was evaporated under high vacuum at $50^{\circ} \mathrm{C}$. The residue was washed twice with ethyl ether and the crude product was purified by column chromatography (silanized Silicagel Merck, $\left.\mathrm{CH}_{3} \mathrm{CN} / \mathrm{H}_{2} \mathrm{O}, 3 / 7\right)$. The fractions containing the product were pooled and evaporated under vacuum. The residue was washed with ethyl ether and dried under vacuum $(0.5 \mathrm{~g})$.

\section{MIC Determination}

Minimal inhibitory concentrations (MIC) were determined by microbroth dilution methodology. Inocula were approximately $5 \times 10^{5} \mathrm{CFU} / \mathrm{ml}$. All microorganisms were cultured at $37^{\circ} \mathrm{C}$. MIC were read at $18 \sim 24$ hours, except for Neisseria gonorrhoeae, Haemophilus influenzae, and Bacteroides fragilis (48 hours). $N$. gonorrhoeae and $H$. influenzae were incubated in a $5 \%$ $\mathrm{CO}_{2}$ atmosphere. B. fragilis was incubated in an anaerobic gas mixture. Media used were: Oxoid Iso-sensitest broth for staphylococci, E. faecalis, and Gram-negative bacteria; Difco Todd-Hewitt broth for streptococci; Difco GC base broth with 1\% BBL Iso-Vitalex for $N$. gonorrhoeae, and Difco Brain-Hearth Infusion broth with $1 \%$ Difco supplement $\mathrm{C}$ for $H$. influenzae.

\section{Results}

\section{Fermentation}

Fermentation of strain $3 / \mathrm{W}$ was performed in a fermentor containing 8 liters of medium $\mathrm{T} / 2$ as described in Experimental. When the time course of antibiotic was prolonged to 275 hours, the HPLC chromatogram showed, besides teicoplanin complex, the presence of a new complex at higher retention time (Fig. 1). The time course of antibiotic production (Fig. 2) indicates that the formation of teicoplanin reaches a maximum at 150 hours and then decreases, and the formation of the second complex steadily increases up to 275 hours.

\section{Recovery and Purification}

The fermentation broth was brought to $\mathrm{pH} 11$ with concentrated $\mathrm{NaOH}$, filtered on Clarcel and the filtrate brought to $\mathrm{pH} 7.8$ with $10 \% \mathrm{HCl}$, and adsorbed on the polyamide resin. Fractions containing the new complex, obtained by the elution described in Experimental, were fractionated by the Amberlite XAD-7 column chromatography, yielding $1.3 \mathrm{~g}$ of crude material. A portion of this material $(500 \mathrm{mg})$ was submitted to the affinity chromatography. The pooled fractions containing the new complex yielded, after ultrafiltration and lyophilization, $85 \mathrm{mg}$ of pure product, named teico 2 . 
Fig. 1. HPLC of the fermentation broth after 275 hours.

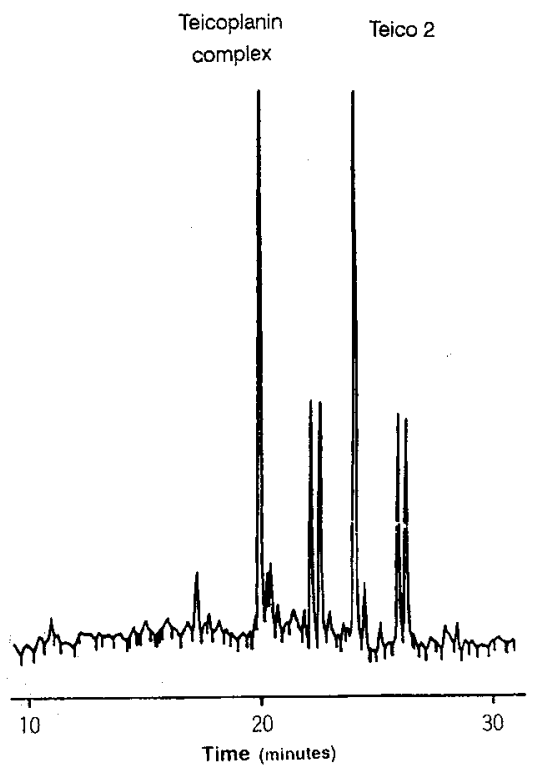

Fig. 2. Time course of the antibiotic production.

$\triangle$ pmv, $\square$ teicoplanin complex, $\bigcirc$ teico 2 .

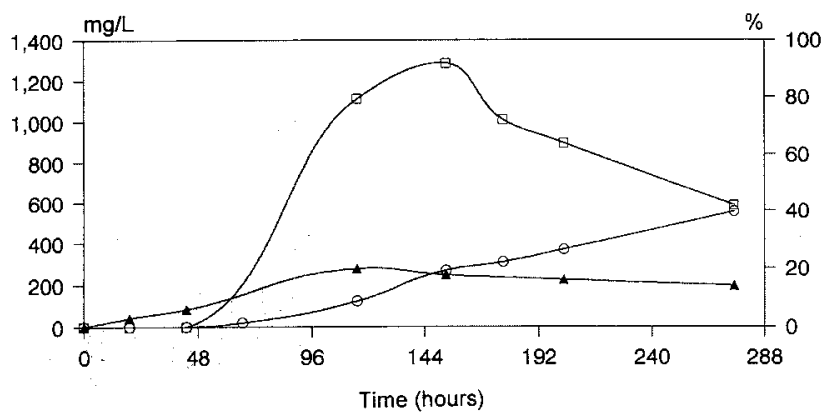

Structure Determination

Spectroscopic Properties of Teico 2 Complex

The HPLC chromatogram of teico 2 (Fig. 3) strongly suggests that it is a complex formed by components similar to those of teicoplanin and differing somewhere in the molecule. The structure was then determined on the basis of the acquired knowledge on the relationships between structure and physicochemical characteristics in the field of glycopeptides ${ }^{5 \sim 9)}$.

The FAB-MS spectrum of teico 2 (Fig. 4) shows several peaks corresponding to a mixture of compounds, each having a molecular weight one unit lower than the corresponding component of the teicoplanin complex ${ }^{6}$. These findings strongly suggest that the number of nitrogen atoms in teico 2 must be different from that of teicoplanin, whereas the fatty acid chains appear unchanged. The whole picture of all the relevant ions
Fig. 3. HPLC of teico 2 complex.

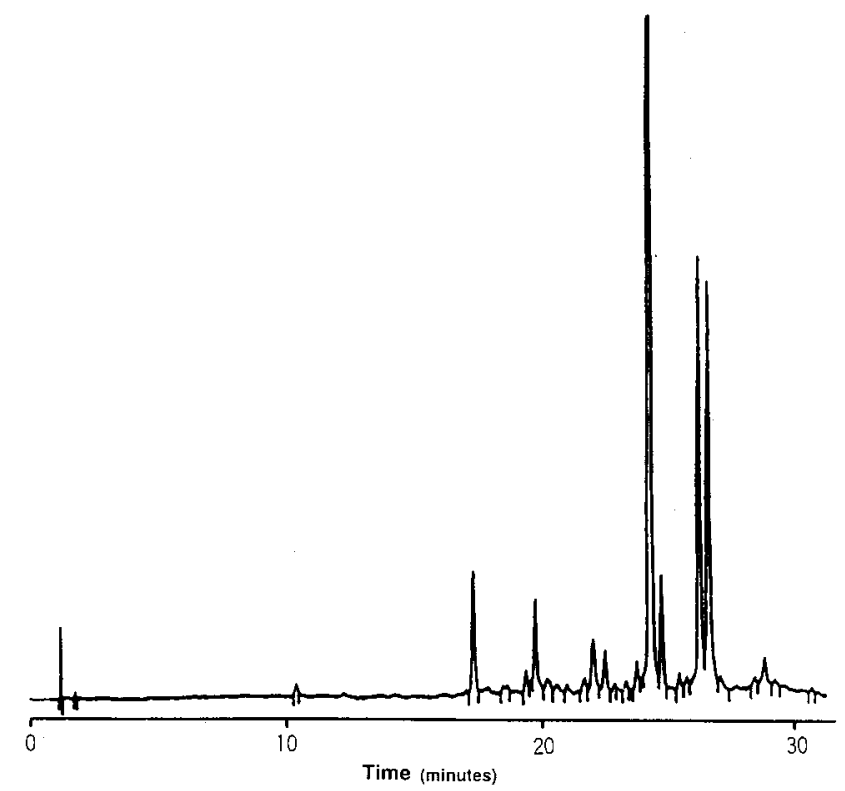

Fig. 4. Positive ion FAB-MS spectrum (top) and expanded quasi-molecular ions region (bottom) of teico 2 complex.

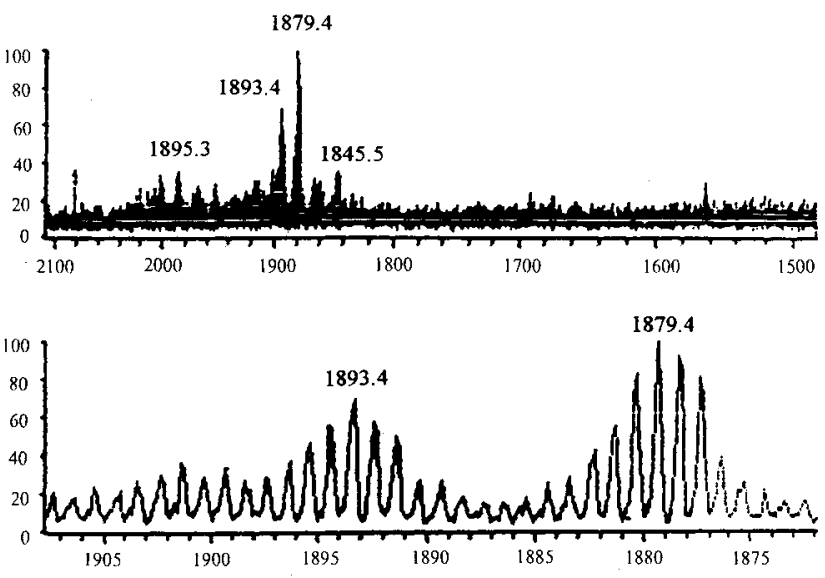

observable is reported in Table 1 together with the assignments.

The general appearance of the proton NMR spectrum of teico 2 corresponds to that of teicoplanin ${ }^{5 \sim 8)}$ and the minor but significant differences emerged after the complete attribution are shown in Table 2 . The variation of the structure of teico 2 resides in the right-hand side of the molecule, namely in the amino acid 1 , as indicated by the absence of the signal of $\mathrm{x} 1$ and by the differences in the signals of $x 2,1 b, 1 e$, and $1 f$. Thus, the combined results of MS and NMR analyses suggest that the difference in structure between teico 2 and teicoplanin is due to the presence of a keto group instead of the $\mathrm{CH}-\mathrm{NH}_{2}$ group of amino acid 1. The structure of teico 
Table 1. Ions observed from teico 2 in positive ion Fast Atom Bombardment Mass Spectra.

\begin{tabular}{ccl}
\hline$m / z$ & Rel. int. & \multicolumn{1}{c}{ Inference } \\
\hline 1999 & 0.1 & $(\mathrm{M}+\mathrm{H}+\mathrm{TG})^{+}$for components 4 and 5 \\
1985 & 0.2 & $(\mathrm{M}+\mathrm{H}+\mathrm{TG})^{+}$for components 2 and 3 \\
1891 & 0.6 & $(\mathrm{M}+\mathrm{H})^{+}$for components 4 and 5 \\
1877 & 1.0 & $(\mathrm{M}+\mathrm{H})^{+}$for components 2 and 3 \\
1875 & 0.1 & $(\mathrm{M}+\mathrm{H}]^{+}$for component 1 and dehydrogenation products from 2 and 3 \\
1857 & 0.1 & $(\mathrm{M}+2 \mathrm{H}-\mathrm{Cl})^{+}$for components 4 and 5 \\
1843 & 0.2 & $(\mathrm{M}+2 \mathrm{H}-\mathrm{Cl})^{+}$for components 2 and 3 \\
\hline
\end{tabular}

$m / z$ refers to the lowest isotope peak of the cluster. $\mathrm{TG}=\alpha$-thioglycerol.

Table 2. Relevant proton NMR data for teico 2 and teicoplanin.

\begin{tabular}{cccc}
\hline Proton & Teico & Teico 2 & Diff. \\
\hline xl & 4.7 & - & \\
x2 & 4.8 & 5.0 & +0.2 \\
x3 & 5.3 & 5.3 & 0 \\
x4 & 5.7 & 5.8 & +0.1 \\
x5 & 4.4 & 4.3 & -0.1 \\
x6 & 4.1 & 4.1 & 0 \\
x7 & 4.4 & 4.5 & +0.1 \\
Ib & 6.6 & 7.0 & +0.4 \\
le & 6.9 & 6.5 & -0.4 \\
If & 7.1 & 7.5 & +0.4 \\
$4 \mathrm{~b}$ & 5.6 & 5.5 & -0.1 \\
$4 \mathrm{f}$ & 5.1 & 5.2 & +0.1 \\
$\mathrm{z6}$ & 5.3 & 5.3 & 0 \\
6b & 7.8 & 7.8 & 0 \\
5f & 6.7 & 6.7 & 0 \\
5e & 6.7 & 6.7 & 0 \\
\hline
\end{tabular}

$\delta$ chemical shift, ppm ( $\delta$ difference) (see Fig. 5 for structure and nomenclature).

2 complex is shown in Figure 5.

\section{Preparation of Teico 2 Aglycone}

Confirmation of the structure was obtained by acid hydrolysis of teico 2 to the single teico 2 aglycone and its spectrometric analysis. Teico $2(50 \mathrm{mg})$ was suspended in $1.5 \mathrm{ml}$ of $\mathrm{CF}_{3} \mathrm{CH}_{2} \mathrm{OH}$ saturated with $\mathrm{HCl}$ and kept in a sealed tube at $80^{\circ} \mathrm{C}$ overnight. The reaction mixture was evaporated to dryness under vacuum, dissolved in $6 \mathrm{ml}$ of a mixture of $\mathrm{H}_{2} \mathrm{O}-\mathrm{CH}_{3} \mathrm{CN}, 3: 1$ and submitted to affinity chromatography ${ }^{1)}$. The fractions containing the aglycone and the pseudoaglycone (HPLC analysis) were pooled and acidified with $\mathrm{HCO}_{2} \mathrm{H}$. The precipitate collected by centrifugation was dried under vacuum $(25 \mathrm{mg})$. Successive separation and purification were obtained by preparative TLC on silanized Silicagel plates Merck RP-8 and 5\% $\mathrm{Na}_{2} \mathrm{SO}_{4}-\mathrm{CH}_{3} \mathrm{CN}, 7: 3$ as mobile phase. The relevant bands were scraped and extracted with $\mathrm{CH}_{3} \mathrm{CN}-\mathrm{H}_{2} \mathrm{O}, 1: 1$. After evaporation under vacuum $14 \mathrm{mg}$ of aglycone and $6 \mathrm{mg}$ of pseudo-
Fig. 5. Structure of teico 2 complex.

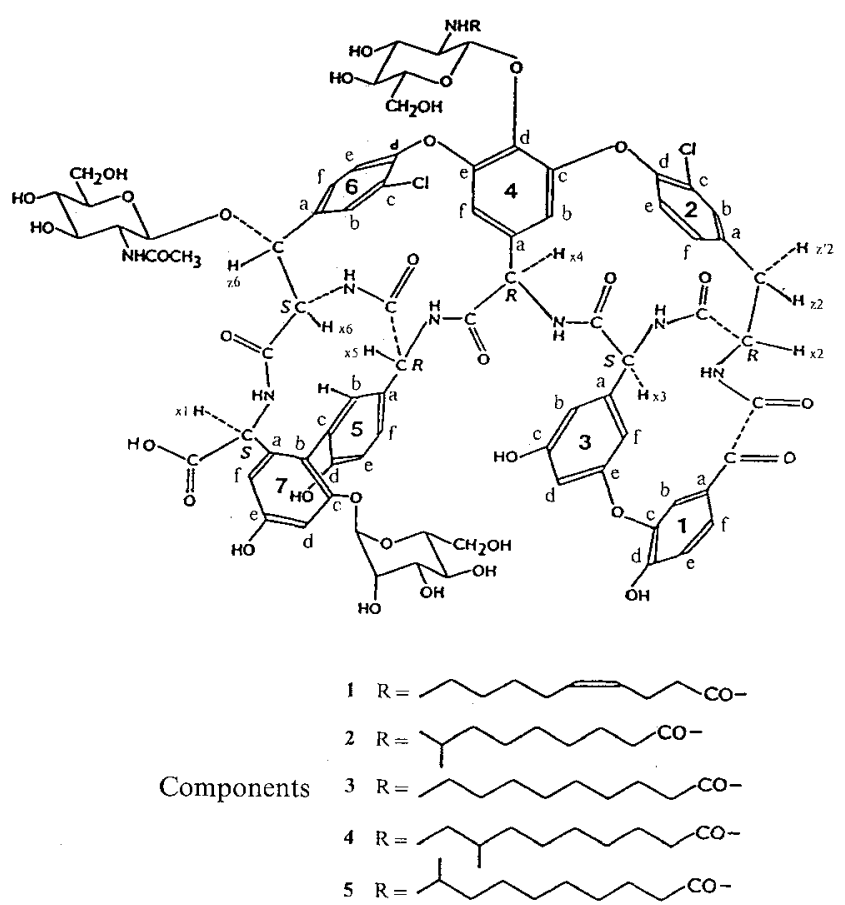

Configuration ( $R$ or $S$ ) of the asymmetric centers and nomenclature proposed by D. H. WILLIAMs for teicoplanin ${ }^{6)}$.

aglycone were obtained.

\section{Spectroscopic Properties of Teico 2 Aglycone}

The various peaks present in the FAB spectrum of teico 2 aglycone (Fig. 6) correspond to the protonated molecule $(m / z 1197)$ and to the adduct ions with sodium $(m / z 1219)$, potassium $(m / z 1235)$, and cesium $(m / z 1329)$ of the aglycone of teico 2.

The proton NMR spectrum of teico 2 aglycone (Fig. 7) allowed the complete attribution of all the signals (displayed in Table 3 in comparison with the signals of teicoplanin aglycone) and confirmed the structure.

The UV spectra of teico 2 aglycone at various $\mathrm{pH}$ values confirm the structure attribution (Table 4). In fact, in addition to the absorption typical of teicoplanin 
Fig. 6. Positive ion FAB-MS spectrum (top) and expanded quasi-molecular ions region (bottom) of teico 2 aglycone.
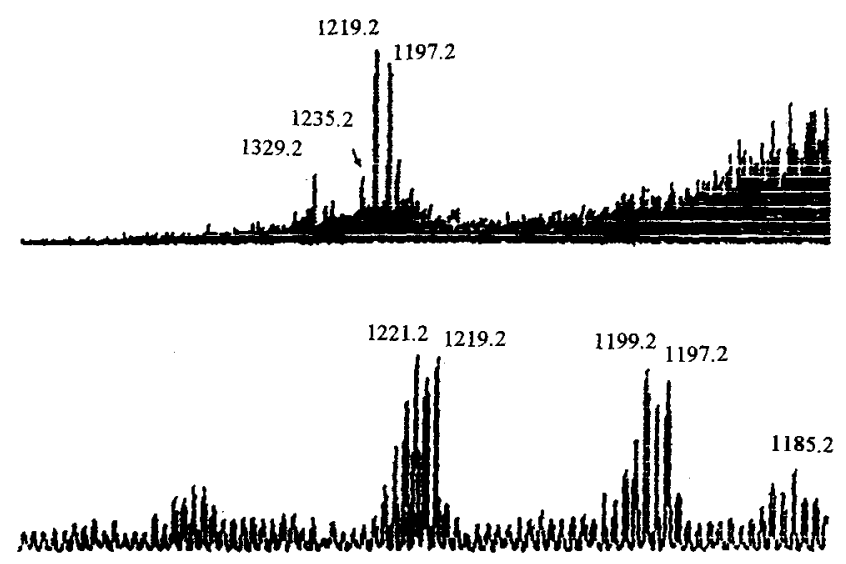

Fig. 7. Proton NMR spectrum of teico 2 aglycone at $250 \mathrm{MHz}$

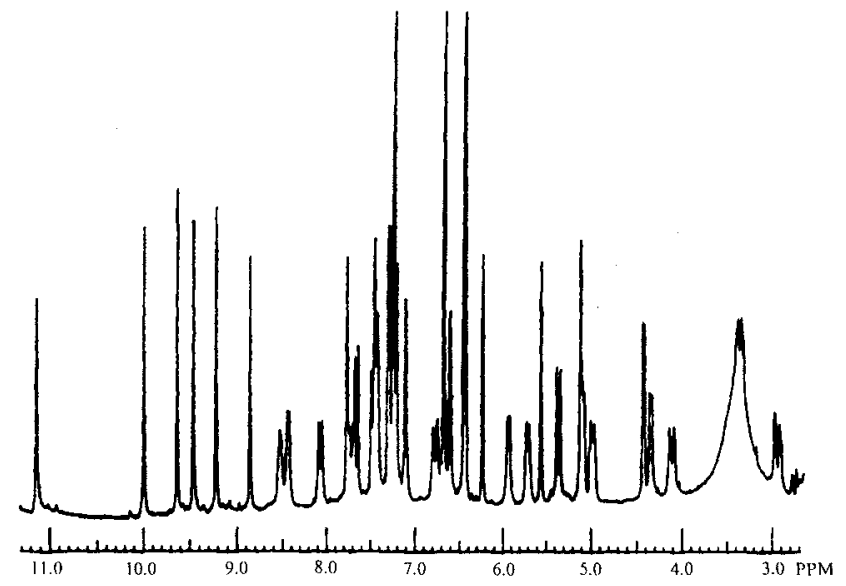

(maximum at about $280 \mathrm{~nm}$ in acidic solution, shifting to about $300 \mathrm{~nm}$ in basic solution) they show in acidic solution a low intensity shoulder at $320 \mathrm{~nm}$ and in basic solution a high intensity maximum at $368 \mathrm{~nm}$. The shoulder is attributed to the $n \rightarrow \pi^{*}$ transition of the additional $\mathrm{C}=\mathrm{O}$ group, while from the latter maximum the presence of an ionizable group related to the chromophore with a pKa value of 6.3 can be deduced. This behaviour is interpreted as due to the $\alpha$-keto-amide group which is in conjugation with the $\pi$ system of the aromatic ring 1 and which produces a vinylogous acid with the para hydroxyl group $^{10}$ ). This new maximum at $368 \mathrm{~nm}$ in basic solution reflects a new chromophore connected with an acidic ionizable function, which, for the sake of speculation, can be depicted as follows:
Table 3. Relevant proton NMR data for teico 2 aglycone in comparison with teicoplanin aglycone ${ }^{7)}$.

\begin{tabular}{|c|c|c|}
\hline Proton & Teico aglyc & Teico 2 aglyc \\
\hline $\mathrm{x} 1$ & 5.47 & - \\
\hline $\mathrm{x} 2$ & 4.92 & 5.00 \\
\hline $\mathrm{x} 3$ & 5.35 & 5.37 \\
\hline$x 4$ & 5.60 & 5.66 \\
\hline x5 & 4.33 & 4.35 \\
\hline$x 6$ & 4.10 & 4.11 \\
\hline$x 7$ & 4.42 & 4.45 \\
\hline wl & 8.53 & - \\
\hline w2 & 8.10 & 8.06 \\
\hline w3 & 7.66 & 7.70 \\
\hline w4 & 7.53 & 7.40 \\
\hline w5 & 8.38 & 8.51 \\
\hline w6 & 6.62 & 6.76 \\
\hline w7 & 8.40 & 8.42 \\
\hline $\mathrm{OH}-\mathrm{z6}$ & 5.85 & 5.86 \\
\hline $\mathrm{OH}-1$ & 9.88 & 9.87 \\
\hline $\mathrm{OH}-3$ & 9.63 & 11.00 \\
\hline $\mathrm{OH}-4$ & 9.45 & 9.47 \\
\hline $\mathrm{OH}-5$ & 9.08 & 9.06 \\
\hline $\mathrm{OH}-7 \mathrm{c}$ & 8.78 & 8.71 \\
\hline $\mathrm{OH}-7 \mathrm{e}$ & 9.34 & 9.34 \\
\hline $\mathrm{z} 2$ & 2.8 & 2.93 \\
\hline$z^{\prime} 2$ & 3.35 & 3.37 \\
\hline $\mathrm{z} 6$ & 5.10 & 5.10 \\
\hline $\mathrm{lb}$ & 6.77 & 7.20 \\
\hline le & 7.02 & 7.20 \\
\hline if & 7.20 & 7.20 \\
\hline $2 b$ & 7.20 & 7.20 \\
\hline $2 \mathrm{e}$ & 7.16 & 7.26 \\
\hline $2 \mathrm{f}$ & 7.86 & 7.64 \\
\hline $4 b$ & 5.50 & 5.59 \\
\hline $4 f$ & 5.08 & 5.14 \\
\hline $5 b$ & 7.07 & 7.11 \\
\hline $5 e$ & 6.65 & 6.68 \\
\hline $5 \mathrm{f}$ & 6.68 & 6.68 \\
\hline $6 \mathrm{~b}$ & 7.77 & 7.77 \\
\hline $6 e$ & 7.19 & 7.20 \\
\hline $6 \mathrm{f}$ & 7.43 & 7.45 \\
\hline $7 d$ & 6.39 & 6.44 \\
\hline $7 \mathrm{f}$ & 6.24 & 6.22 \\
\hline
\end{tabular}

$\delta$ chemical shift, ppm (see Fig. 5 for structure and nomenclature).

Table 4. UV data for teico 2 aglycone in aqueous solutions at various $\mathrm{pH}$ values.

\begin{tabular}{lccccc}
\hline $\mathrm{pH}$ of the solution & $\lambda_{\text {max }}, \mathrm{nm}$ & $\varepsilon_{\text {mol }}$ & $\lambda_{\text {max }}, \mathrm{nm}$ & $\varepsilon_{\text {mol }}$ \\
\hline $0.1 \mathrm{~N} \mathrm{HCl}$ and 4.7 & 285 & 15,360 & $320 \mathrm{sh}$ & 5,940 \\
6.1 & 280 & 14,700 & 365 & 6,860 \\
7.4 & 276 & 15,960 & 368 & 16,660 \\
8.5 & 276 & 17,914 & 368 & 17,300 \\
$0.1 \mathrm{~N} \mathrm{KOH}$ & 298 & 21,700 & 356 & 16,240 \\
\hline
\end{tabular}

$\mathrm{sh}=$ shoulder

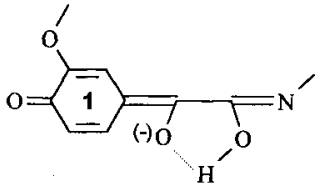


Table 5. In vitro activity (MIC, $\mu \mathrm{g} / \mathrm{ml}$ ) of teico 2 complex in comparison with teicoplanin.

\begin{tabular}{lcc}
\hline & Teico 2 & Teicoplanin \\
\hline Staphylococcus aureus Tour L165 & 4 & 0.5 \\
S. aureus Tour 30\% bovine serum & 4 & 0.5 \\
S. aureus clinical isolate L561 & $>128$ & 8 \\
S. epidermidis ATCC12228 L147 & 128 & 0.5 \\
S. epidermidis clinical isolate L533 & $>128$ & 8 \\
S. haemolyticus clinical isolate L602 & $>128$ & 16 \\
Streptococcus pyogenes C203 L49 & 0.125 & 0.063 \\
S. pneumoniae UC41 L44 & 0.125 & 0.063 \\
Enterococcus faecalis ATCC7080 L149 & 2 & 0.125 \\
E. faecalis clinical isolate L562 & $>128$ & 64 \\
Escherichia coli SKF12140 L47 & $>128$ & $>128$ \\
Pseudomonas aeruginosa ATCC10145 L4 & $>128$ & $>128$ \\
Proteus vulgaris X19H ATCC881 L79 & $>128$ & $>128$ \\
\hline
\end{tabular}

Table 6. In vitro activity (MIC, $\mu \mathrm{g} / \mathrm{ml}$ ) of teico 2 aglycone in comparison with teicoplanin aglycone and teicoplanin.

\begin{tabular}{lccc}
\hline & $\begin{array}{c}\text { Teico } 2 \\
\text { aglycone }\end{array}$ & $\begin{array}{c}\text { Teicoplanin } \\
\text { aglycone }\end{array}$ & Teicoplanin \\
\hline Staphylococcus aureus Tour L165 & 0.13 & 0.063 & 0.25 \\
S. aureus Tour 30\% bovine serum & 16 & 0.25 & 1 \\
S. aureus clinical isolate L561 & 0.5 & - & 16 \\
S. epidermidis ATCC12228 L147 & 0.13 & 0.016 & 1 \\
S. epidermidis clinical isolate L533 & 0.13 & - & 32 \\
S. haemolyticus clinical isolate L602 & 4 & 0.25 & 16 \\
Streptococcus pyogenes C203 L49 & 1 & 0.125 & 0.06 \\
S. pneumoniae UC41 L44 & 0.5 & 0.125 & 0.13 \\
Enterococcus faecalis ATCC7080 L149 & 2 & -125 & 0.13 \\
E. faecalis clinical isolate L562 & $>128$ & - & $>128$ \\
Bacteroides fragilis ATCC23745 L1010 & $>128$ & 8 & 32 \\
Neisseria gonorrioeae ISM68/126 L997 & 64 & 16 & $>128$ \\
Haemophylus influenzae type b ATCC19418 L970 & 32 & 64 & $>128$ \\
Escherichia coli SKF12140 L47 & $>128$ & $>128$ \\
Pseudomonas aeruginosa ATCC10145 L4 & $>128$ & 128 & $>128$ \\
Proteus vulgaris X19H ATCC881 L79 & $>128$ & & \\
\hline
\end{tabular}

\section{Synthesis of Teico 2 Aglycone}

As a final confirmation of the structure, the synthesis of teico 2 aglycone was carried on starting from teicoplanin aglycone. The method described for ristocetin was essentially applied ${ }^{11)}$ (see Experimental). From $3 \mathrm{~g}$ of teicoplanin aglycone $0.5 \mathrm{~g}$ of teico 2 aglycone was obtained, whose analytical characteristics resulted identical to those of the product obtained by fermentation.

\section{Microbiological Activity}

Teico 2 complex (Table 5) and teico 2 aglycone (Table 6) show a microbiological activity similar but somewhat lower than that of teicoplanin complex and teicoplanin aglycone.

\section{Discussion}

The physico-chemical data on teico 2 aglycone and the unequivocal synthesis starting from teicoplanin aglycone clearly demonstrate that the structure corresponds to the substitution of a carbonyl group for the $\mathrm{CH}-\mathrm{NH}_{2}$ group of amino acid 1 (Fig. 5). These data are in accordance with those reported in literature for similar glycopeptide derivatives, produced either by microorganisms ${ }^{10,12)}$ or by chemical reaction ${ }^{11)}$.

The MS-FAB of teico 2 complex, showing that the single components differ from the corresponding members of teicoplanin by one mass unit less, indicates that no other alterations are present. This is agreement with the findings of NMR spectroscopy, showing that the sugars are the same and the fatty acid moieties present a similar pattern.

From the structure and the time course of fermentation, in which the production of teico 2 is accompanied 
by a decrease in teicoplanin concentration, it can be assumed that the former is a transformation product of the latter, due to a simple transamination reaction.

\section{References}

1) CoRti, A. \& G. CASSANI: Synthesis and characterization of D-alanyl-D-alanine-agarose: a new bioselective adsorbent for affinity chromatography of glycopeptide antibiotics. Applied Biochem. Biotech. 11: $101 \sim 109$, 1985

2) VÉKEY, K.: Calibration in positive and negative ion fast atom bombardment using salt mixtures. Org. Mass Spectrom. 24: 183 185, 1989

3) MARION, D. \& K. WÜTHRICH: Application of phase sensitive two-dimensional correlated spectroscopy (COSY) for measurement of ${ }^{1} \mathrm{H}^{-1} \mathrm{H}$ spin-spin constants in proteins. Biochemical and Biophysical Research Communications 113: $967 \sim 974,1983$

4) Gray, A. R. \& R. C. Fuson: The highly activated carbonyl group: mesitylglyoxal. J. Am. Chem. Soc. 56: $739 \sim 741,1934$

5) Hunt, A. H.; R. M. Molloy, J. L. Occolowitz, G. G. MARConi \& M. Debono: Structure of the major glycopeptide of the teicoplanin complex. J. Am. Chem. Soc. 106: $4891 \sim 4895,1984$

6) Barna, J. C. J.; D. H. Williams, D. J. M. Stone, T.
W. Christina Leung \& D. M. Doddrell: Structure elucidation of the teicoplanin antibiotics. J. Am. Chem. Soc. 106: 4895 4902, 1984

7) Malabarba, A.; P. Ferrari, G. G. Gallo, J. Kettenring \& B. Cavalleri: Teicoplanin, antibiotics from Actinoplanes teichomyceticus nov. sp. VII. Preparation and NMR characteristics of the aglycone of teicoplanin. $\mathbf{J}$. Antibiotics 39: $1430 \sim 1442,1986$

8) Heald, S. L.; L. Mueller \& P. W. JefFs: Structural analysis of teicoplanin A2 by 2D NMR. J. Magnetic Resonance 72: 120 138, 1987

9) Trani, A.; P. Ferrari, R. Pallanza \& G. Tarzia: Deamino teicoplanin and its derivatives. Synthesis, antibacterial activity, and binding strength to Ac-D-AlaD-Ala. J. Med. Chem. 32: 310 314, 1989

10) Skelton, N. J. \& D. H. Williams: Structure elucidation of the novel glycopeptide antibiotic UK-68,597. J. Org. Chem. 55: 3718 3723, 1990

11) Herrin, T. R.; A. M. Thomas, T. J. Perun, J. C. Mao \& S. W. Fesik: Preparation of biologically active ristocetin derivatives: replacements of the $\mathbf{1}^{\prime}$-amino group. J. Med. Chem. 28: $1371 \sim 1375,1985$

12) Boeck, L. D.; G. M. Clem, C. L. Hershberger, M. T. ANDERSON \& K. H. MrChEL: New glycopeptide antibiotic prepared by co-fermentation of Actinoplanes missouriensis strains CSV558 and CUC014. UK Patent: GB 2148 303-A, 1985 